\title{
HLA-B Antigen Type
}

National Cancer Institute

\section{Source}

National Cancer Institute. HLA-B Antigen Type. NCI Thesaurus. Code C128957.

The determination of the amount of human leukocyte antigen, class I, group B (HLA-B), present in a sample. 